\title{
THE INFLUENCES OF ADHERENCE TO TAMOXIFEN AND CYP2D6 PHARMACOGENETICS ON PLASMA CONCENTRATIONS OF THE ACTIVE METABOLITE (Z)-ENDOXIFEN IN BREAST CANCER
}

\section{José Claudio Casali da Rocha'}

${ }^{1}$ Hospital Erasto Gaertner - Curitiba (PR), Brazil.

Tamoxifen efficacy in breast cancer is suspected to depend on adherence and intact drug metabolism. We evaluated the role of adherence behavior and pharmacogenetics on the formation rate of (Z)-endoxifen. In 192 Brazilian patients, we assessed plasma levels of tamoxifen and its metabolites at 3, 6, and 12 months of treatment (LC-MS/MS), adherence behavior (Morisky Medication Adherence Scale), and CYP2D6 and other pharmacogene polymorphisms (MALDI-TOF mass spectrometry and real-time PCR). Adherence explained $47 \%$ of the variability of tamoxifen plasma concentrations $(\mathrm{p}<0.001)$. While CYP2D6 alone explained $26.4 \%$, the combination with adherence explained $40 \%$ of $(\mathrm{Z})$-endoxifen variability at 12 months $(\mathrm{p}<0.001)$. The influence of low adherence not to achieving relevant $(\mathrm{Z})$-endoxifen levels was the highest in patients with non-compromised CYP2D6 function (RR 3.65, 95\%CI 1.48-8.99). As a proof-of-concept, we demonstrated that (Z)-endoxifen levels are influenced by patient adherence to both tamoxifen and CYP2D6, which is particularly relevant for patients with full CYP2D6 function.

Keywords: Tamoxifen; Breast Cancer; CYP2D6; Adherence; Endoxifen. 